\title{
Effects of NVP-BEZ235, a dual phosphatidylinositol 3-kinase/mammalian target of rapamycin inhibitor, on HTLV-1-infected T-cell lines
}

\author{
CHIE ISHIKAWA ${ }^{1,2}$, MASACHIKA SENBA $^{3}$ and NAOKI MORI ${ }^{2}$ \\ ${ }^{1}$ Division of Health Sciences, Transdisciplinary Research Organization for Subtropics and Island Studies, \\ University of The Ryukyus, Nishihara, Okinawa 903-0213; ${ }^{2}$ Department of Microbiology and Oncology, \\ Graduate School of Medicine, University of The Ryukyus, Nishihara, Okinawa 903-0215; \\ ${ }^{3}$ Department of Pathology, Institute of Tropical Medicine, Nagasaki University, Nagasaki 852-8523, Japan
}

Received December 21, 2016; Accepted January 29, 2018

DOI: $10.3892 / \mathrm{ol} .2018 .7979$

\begin{abstract}
Adult T-cell leukemia (ATL) is an aggressive type of malignancy caused by human T-cell leukemia virus type 1 (HTLV-1). In ATL, the phosphatidylinositol 3-kinase (PI3K)/Akt/mammalian target of rapamycin (mTOR) signaling pathway is constitutively active, promoting cell proliferation, survival and chemoresistance. Thus, the PI3K signaling pathway is an attractive therapeutic target for ATL. In the present study, the effects of RAD001 (an mTOR inhibitor), NVP-BKM120 (a pan-PI3K inhibitor) and NVP-BEZ235 (a novel dual PI3K/mTOR inhibitor) on cultured HTLV-1-infected T-cell lines were compared. The results demonstrated that NVP-BEZ235 was more efficacious compared with RAD001 and NVP-BKM120 at inhibiting cell growth. NVP-BEZ235 exhibited cytostatic rather than cytotoxic effects on various HTLV-1-infected T-cell lines, where it induced cell cycle arrest at $\mathrm{G}_{1}$ phase. NVP-BEZ235 downregulated cyclin D1, cyclin D2, cyclin E, cyclin dependent kinase (CDK)2 and CDK4 expression, and the phosphorylation of retinoblastoma protein. In C.B-17/Icr-severe combined immune deficiency mice implanted with HTLV-1-infected HUT-102 cells, oral NVP-BEZ235 caused marked retardation of tumor growth compared with the control. The present in vitro and
\end{abstract}

Correspondence to: $\mathrm{Dr}$ Chie Ishikawa, Division of Health Sciences, Transdisciplinary Research Organization for Subtropics and Island Studies, University of The Ryukyus, 1 Senbaru, Nishihara, Okinawa 903-0213, Japan

E-mail: chiezo@lab.u-ryukyu.ac.jp

Professor Naoki Mori, Department of Microbiology and Oncology, Graduate School of Medicine, University of The Ryukyus, 207 Uehara, Nishihara, Okinawa 903-0215, Japan

E-mail: naokimori50@gmail.com

Key words: adult T-cell leukemia, NVP-BEZ235, NVP-BKM120, RAD001, human T-cell leukemia virus type 1, phosphatidylinositol 3-kinase, mammalian target of rapamycin, Akt in vivo studies highlight the efficacious dual inhibition of PI3K, and mTOR following NVP-BEZ235 treatment. Thus, the results of the current study provide preclinical rationale for phase I clinical studies to examine the effects of NVP-BEZ235 in patients with ATL.

\section{Introduction}

Adult T-cell leukemia (ATL) caused by the clonal proliferation of human T-cell leukemia virus type 1 (HTLV-1)-infected T cells (1) is highly resistant to currently available chemotherapies, and its prognosis is the worst among common subtypes of mature T/natural killer-cell neoplasms (2). Except for allogenic hematopoietic stem cell transplantation for some patients with ATL, no reliable treatment has yet been established (3).

Constitutive activation of phosphatidylinositol 3-kinase (PI3K) is linked to oncogenesis and disease progression in a variety of solid tumors and hematologic malignancies including ATL, apparently conferring drug resistance and resulting in poor prognosis (4). PI3K stimulates the production of phosphatidylinositol 3,4,5-triphosphate and subsequently the activation of 3-phosphoinositide-dependent protein kinase 1 (PDK1)/Akt and mammalian target of rapamycin (mTOR). This pathway integrates various extracellular stimuli to regulate several cellular processes, such as mRNA translation, cell survival and cell cycle progression (5). The inactive phosphorylated form of phosphatase and tensin homologue (PTEN), a negative PI3K regulator, are often expressed on ATL cells and contribute to constitutive PI3K signaling (6). These pathways could be suitable targets for new therapies for ATL. Evidence suggests that PI3K-selective targeting can potentially suppress this pathway. In this regard, several types of PI3K inhibitors are currently being investigated (7), including, NVP-BKM120, which is reported to be effective against several types of malignancies in cell- (4,8-10) and laboratory animal-based studies (11).

On the other hand, allosteric mTOR inhibitors, such as rapamycin and its analogues (temsirolimus and everolimus; earlier code name RAD001), mainly target mTOR complex 1 (mTORC1), and the former are approved for use 
in various cancers (12). However, the mTORC1-specificity of rapamycin analogues can limit their efficacies based on the feedback activation of upstream PI3K signaling, resulting in Akt hyperactivation through mTORC2 (13). Targeting the $\mathrm{PI} 3 \mathrm{~K} / \mathrm{Akt} / \mathrm{mTOR}$ pathway at multiple levels may therefore provide a more effective antitumor activity than selective inhibition of only one component of the pathway. Unlike rapamycin and its analogues, the new generations of dual PI3K/mTOR inhibitors competitively inhibit ATP and target the catalytic site of both kinases (7). These inhibitors have more potent antitumor effects and can suppress the prosurvival regulatory feedback (14). Oral NVP-BEZ235 is reported to inhibit the kinase activities of mTORC1, mTORC2 and PI3K (15). Several pre-clinical studies reported the efficacy of NVP-BEZ235 in various lymphoid malignancies $(14,16-18)$ and its effects, either alone or in combination with other compounds, are currently being tested in the treatment of various other malignancies (12).

The aim of the present study was to compare the effects of NVP-BEZ235 with those of NVP-BKM120 (pan-class I PI3K inhibitor) and RAD001 (mTORC1 inhibitor) at a cellular level using HTLV-1-infected $\mathrm{T}$ cells. In addition, we also tested the effects of NVP-BEZ235 in laboratory animals harboring ATL tumors. The results suggest that suppression of both PI3K and mTORC2, in addition to mTORC1, is more efficacious in the treatment of ATL, compared with inhibition of each of these kinases.

\section{Materials and methods}

Inhibitors. NVP-BEZ235, NVP-BKM120 and RAD001 were kindly gifted by Novartis Institutes for BioMedical Research (Basel, Switzerland). Each was dissolved in dimethyl sulfoxide (cat. no. 13407-45; Nacalai Tesque, Inc., Kyoto, Japan) to create stock solution that was stored at $-80^{\circ} \mathrm{C}$.

Cell culture. HTLV-1-transformed T-cell lines, MT-2, MT-4, HUT-102 and SLB-1, and ATL-derived T-cell lines, TL-OmI and ED-40515(-), were maintained in RPMI-1640 medium (cat. no. 30264-56; Nacalai Tesque, Inc.) supplemented with $10 \%$ heat-inactivated fetal bovine serum (Biological Industries, Kibbutz Beit Haemek, Israel) and 1\% penicillin/streptomycin (cat. no. 09367-34; Nacalai Tesque, Inc.).

Assessment of cell proliferation and cytotoxicity. Cell proliferation and cytotoxicity were measured by the water-soluble tetrazolium (WST)-8 assay (cat. no. 07553-44; Nacalai Tesque, Inc.). The WST- 8 is taken up by viable cells and reduced to a colored formazan product by mitochondrial dehydrogenase (19). For the WST-8 assay, cells were treated with increasing concentrations of the test drug for $48 \mathrm{~h}$. Then, WST- 8 was added to each well and incubated. Absorbance was measured at $450 \mathrm{~nm}$ by a $680 \mathrm{XR}$ microplate absorbance reader (Bio-Rad Laboratories, Inc., Hercules, CA, USA). All experiments were conducted in triplicates. The drug concentration required for $50 \%$ inhibition of cell proliferation $\left(\mathrm{IC}_{50}\right)$ was evaluated with CalcuSyn software (version 2.0; Biosoft, Cambridge, UK).

Cell cycle analysis. Cell cycle was analyzed using the CycleTEST Plus DNA Reagent kit (cat. no. 340242; Becton-Dickinson
Immunocytometry Systems, San Jose, CA, USA) and an Epics XL flow cytometer (Beckman Coulter, Inc., Brea, CA, USA). The percentages of cells in each cell cycle phase were calculated using MultiCycle software (version 3.0; Phoenix Flow Systems, San Diego, CA, USA).

Apoptosis assay. Apoptosis was quantified using the APO2.7 assay. Cells were stained with phycoerythrin-conjugated APO2.7 antibody (cat. no. IM2088; Beckman Coulter, Marseille, France). This antibody is reported to specifically detect a $38-\mathrm{kDa}$ mitochondrial membrane antigen (7A6), which is expressed during programmed cell death (20). Events were acquired using a flow cytometer.

Western blot analysis. After drug treatment, cells were harvested and lysed in a buffer solution [composition: 62.5 mM Tris-HCl (pH 6.8) (cat. no. 35434-21; Nacalai Tesque, Inc.), $2 \%$ sodium dodecyl sulfate (cat. no. 31607-65; Nacalai Tesque, Inc.), 10\% glycerol (cat. no. 17045-65; Nacalai Tesque, Inc.), 6\% 2-mercaptoethanol (cat. no. 21438-82; Nacalai Tesque, Inc.) and 0.01\% bromophenol blue (cat. no. 021-02911; Wako Pure Chemical Industries, Osaka, Japan)]. The cell lysates (20 $\mu \mathrm{g} /$ lane) were electrophoresed on sodium dodecyl sulfate-polyacrylamide gels and transferred to polyvinylidene difluoride membranes (cat. no. IPVH00010EMD; Merck KGaA, Darmstadt, Germany). The membranes were immunoblotted with the following primary antibodies: Total Akt (cat. no. 9272), phospho-Akt (Ser473; cat. no. 4060), phospho-Akt (Thr308; cat. no. 13038), phospho-PDK1 (Ser241; cat. no. 3061), phospho-p70 S6 kinase (S6K) (Thr421 and Ser424; cat. no. 9204) and phospho-4E-BP1 (Thr70; cat. no. 9455) were all from Cell Signaling Technology, Inc. (Beverly, MA, USA); cyclin D2 (cat. no. sc-593) was from Santa Cruz Biotechnology, Inc. (Santa Cruz, CA, USA); cyclin D1 (cat. no. K0062-3) and phospho-retinoblastoma protein (pRb) (Ser780; cat. no. M054-3S) were from Medical \& Biological Laboratories, Co., (Aichi, Japan); cyclin E (cat. no. MS-870), cyclin dependent kinase (CDK)2 (cat. no. MS-617), CDK4 (cat. no. MS-299), CDK6 (cat. no. MS-398) and actin (cat. no. MS-1295) were from Neomarkers, Inc. (Fremont, CA, USA). Then, the membranes were incubated with species-specific horseradish peroxidase-conjugated secondary antibody (cat. nos. 7074 and 7076) from Cell Signaling Technology, Inc.

Xenograft tumor model. In these experiments, 5-week-old female C.B-17/Icr-severe combined immune deficiency (SCID) mice (Kyudo, Co., Tosu, Japan) were kept in specific pathogen-free conditions, housed in air-conditioned rooms (temperature, $24^{\circ} \mathrm{C}$; humidity, $60 \%$ ) set at $12 / 12 \mathrm{~h} \mathrm{light/dark}$ cycles, and fed standard rodent diet (CE-2 from CLEA Japan, Inc., Tokyo, Japan) and provided with water ad libitum. A total of $1 \times 10^{7}$ HUT-102 cells (suspended in $300 \mu \mathrm{l}$ of RPMI-1640 medium) were injected subcutaneously behind the ears. The tumor-bearing mice were divided at random into two treatment groups ( $\mathrm{n}=6$ each). NVP-BEZ235 was solubilized in $0.5 \%$ methylcellulose and administrated at $40 \mathrm{mg} / \mathrm{kg}$ body weight/day by oral gavage, and the treatment was continued for 28 days, starting the day after cell injection. The control group was treated with vehicle only. Tumor volume was estimated by measuring tumor diameter once every week with a caliper. 
The ellipsoid volume formula ( $\pi / 6 \mathrm{x}$ length $\mathrm{x}$ width $\mathrm{x}$ height) was used (21). The animals were weighed once every week, and then sacrificed at day 28. This was followed by excision of the tumor for analysis (see below). The present study was approved by the Animal Care and Use Committee (reference no. 5276) and performed according to the Guidelines for Animal Experimentation of our university.

Histopathological examination and terminal deoxynucleotidyl transferase deoxyuridine triphosphate nick end labeling (TUNEL) assay. Tumor tissues were fixed in formalin (Wako Pure Chemical Industries), dehydrated using ethanol (Japan Alcohol Selling Co., Tokyo, Japan) solution and embedded in paraffin (cat. no. 09620; Sakura Finetek Japan Co., Tokyo, Japan). The paraffin-embedded ATL specimens were stained with hematoxylin and eosin (H\&E; cat. nos. 234-12 and 1159350025; Merck KGaA) and examined under a microscope. DNA fragmentation was analyzed by TUNEL kit (cat. no. 11684817910; Roche Applied Science, Penzberg, Germany). Cells were examined under Axioskop-2 Plus light microscope equipped with an Achroplan lens (Zeiss, Hallbergmoos, Germany). Images were acquired with appropriate software (AxioCam 503 color and AxioVision LE64; Zeiss GmbH, Jena, Germany).

Measurements of soluble interleukin-2 receptor (sIL-2R) and soluble cluster of differentiation 30 (sCD30). Using the appropriate enzyme-linked immunosorbent assay (ELISA) kit, we measured serum levels of human sIL-2R (cat. no. DR2A00; R\&D Systems, Inc., Minneapolis, MN, USA) and human sCD30 (cat. no. SK00582-01; Aviscera Bioscience, Inc., Santa Clara, CA, USA), according to the instructions provided by the supplier.

Statistical analysis. All values are expressed as the mean \pm standard deviation (SD). The unpaired t-test was used to test the statistical differences between the two treatment groups. $\mathrm{P}<0.05$ was considered to indicate a statistically significant difference.

\section{Results}

Superior growth inhibitory activity of NVP-BEZ235 compared to NVP-BKM120 and RAD001 in HTLV-1-infected T-cell lines. Considering that PI3K is upstream of mTOR, and is activated in ATL and HTLV-1-infected T-cell lines $(6,22)$, we considered that inhibition of PI3K in addition to mTOR would be a superior strategy to inhibition of mTOR or PI3K alone. We compared the effects of the dual PI3K/mTOR inhibitor NVP-BEZ235 with those of the PI3K inhibitor NVP-BKM120 and the mTOR inhibitor RAD001. To determine the $\mathrm{IC}_{50}$ values, HTLV-1-transformed T-cell lines MT-2, MT-4, HUT-102, SLB-1, and patient-derived ATL cell lines TL-OmI and ED-40515(-) were cultured with increasing concentrations of NVP-BEZ235, NVP-BKM120 and RAD001 (1-1,000 nM) for $48 \mathrm{~h}$ (Fig. 1A-C). Table I shows the $\mathrm{IC}_{50}$ values obtained for NVP-BEZ235 and NVP-BKM120 in HTLV-1-infected T-cell lines. Exposure of these cells to NVP-BEZ235 and NVP-BKM120 inhibited the growth of HTLV-1-infected $\mathrm{T}$-cell lines in a dose-dependent manner with $\mathrm{IC}_{50}$ from
11.9 to $293.0 \mathrm{nM}$ (NVP-BEZ235) and from 397.2 to $2,248.6 \mathrm{nM}$ (NVP-BKM120), as measured by WST-8 assay. Interestingly, lower nanomolar concentrations of NVP-BEZ235 were needed to inhibit the proliferation of HTLV-1-infected T-cell lines compared with NVP-BKM120. RAD001 reduced the proliferation of HTLV-1-infected T-cell lines at low nanomolar concentrations, but increasing doses of RAD001 did not significantly enhance the anti-proliferative activity. The $\mathrm{IC}_{50}$ was not achieved. We also evaluated the effects of the drugs using samples of peripheral blood mononuclear cells (PBMC) obtained from a healthy subject (Fig. 1A-C). NVP-BEZ235, NVP-BKM120 and RAD001 each had minimal effect on PBMC from the healthy donor. Fig. 1D shows the effects of $100 \mathrm{nM}$ of NVP-BEZ235, NVP-BKM120 and RAD001 on cell proliferation in HTLV-1-infected T-cell lines and PBMC from the healthy donor. Cell proliferation in all HTLV-1-infected T-cell lines was most effectively inhibited with NVP-BEZ235. These results indicate that the dual PI $3 \mathrm{~K} / \mathrm{mTOR}$ inhibitor is a more potent suppressor of proliferation of HTLV-1-infected T-cell lines, compared with each compound alone.

NVP-BEZ235 induces cell cycle arrest. For a better understanding of the mechanism of NVP-BEZ235-induced inhibition of HTLV-1-infected T-cell line growth, cell cycle changes were examined (Fig. 2A). We employed propidium iodide staining and DNA content analysis by flow cytometry to determine whether $24 \mathrm{~h}$ of treatment with NVP-BEZ235 altered cell cycle progression. A high percentage of cells in the $G_{0} / G_{1}$ phase of the cell cycle, with a concomitant decrease in the proportion of those in the $\mathrm{S}$ phase, was noted in the presence of NVP-BEZ235, as the concentrations increased. Our findings indicate that NVP-BEZ235 inhibits the growth of HTLV-1-infected T-cell lines by inducing cell cycle arrest at the $\mathrm{G}_{0} / \mathrm{G}_{1}$ phase.

Effects of NVP-BEZ235 on apoptosis. To evaluate whether the anti-ATL activity of NVP-BEZ235 was due to induction of apoptosis, flow cytometric analysis was performed. MT-4 and HUT-102 cells were treated with different concentrations of NVP-BEZ235 for 24-48 $\mathrm{h}$ and apoptosis was assessed by APO2.7 staining. As shown in Fig. 2B, flow cytometric analysis demonstrated a slight increase in apoptotic cells after treatment with NVP-BEZ235, suggesting that the reduction in WST-8 activity was primarily due to inhibition of cell proliferation.

Effects of NVP-BEZ235 on cell cycle regulators. $\mathrm{pRb}$ controls cell cycle progression at the $G_{0} / G_{1}$ to $S$ transition in response to certain signals for growth inhibition (23). To determine the mechanism by which NVP-BEZ235 induces $G_{0} / G_{1}$ cell cycle arrest, the phosphorylation status of $\mathrm{pRb}$ was evaluated by western blot analysis. The level of the phosphorylated form of $\mathrm{pRb}$ was decreased in cells treated with NVP-BEZ235 (Fig. 3A and B). pRb is phosphorylated by catalytic subunits of CDK4/CDK6 and CDK2 complexed with specific regulatory subunits of cyclins D and E, respectively. The expression levels subtypes of D-type cyclins, cyclin D1 and cyclin D2, examined by western blot analysis, were decreased in MT-4, HUT-102 and TL-OmI cells following treatment with NVP-BEZ235. Furthermore, the levels of CDK4 and/or 
CDK6 were also decreased in NVP-BEZ235-treated HUT-102 and TL-OmI cells. Moreover, western blot analysis indicated that treatment with NVP-BEZ235 downregulated the expression of cyclin E and CDK2 in MT-4, HUT-102 and TL-OmI cells (Fig. 3A and B).

NVP-BEZ235 inhibits PI3K/Akt/mTOR pathway. Next, we examined the effects of NVP-BEZ235 on the PI3K/Akt/mTOR signaling pathway. To confirm the efficacy of NVP-BEZ235 against downstream targets of PI3 K and mTOR, we tested the phosphorylation status of the downstream targets of these two kinases, using western blot analysis (Fig. 3C). Treatment with NVP-BEZ235 for 24-48 h induced marked reduction in phosphorylated PDK1 and Akt at Thr308, both targets of the kinase activity of PI3K. Reduced phosphorylation of p70 S6K and 4E-BP1, both targets of mTOR, reflects inhibition of mTOR by NVP-BEZ235. Importantly, the reduced phosphorylation of Akt at Ser473, a feedback phosphorylation substrate of mTORC2, indicates that NVP-BEZ235 also inhibits mTORC2 activity (Fig. 3C). Treatment did not affect total Akt protein level at any dose. Thus, dual inhibition of PI3K and mTOR seems to contribute to decreased cell proliferation by NVP-BEZ235.

Efficacy of NVP-BEZ235 in SCID mouse model of ATL. Mice engrafted with HUT-102 cells were treated daily for 4 weeks with NVP-BEZ235 at $40 \mathrm{mg} / \mathrm{kg} /$ body weight. NVP-BEZ235 resulted in reduction of body weight after 4 weeks of treatment (21.2 vs. $22.3 \mathrm{~g}, \mathrm{P}<0.05)$. A decrease in body weight was observed in NVP-BEZ235-treated mice (maximum loss, 6.5\%) compared with the control mice at week one; however, the growth curve of the NVP-BEZ235 group was similar to that of the control group thereafter. Multiple tumors were not observed in any of the mice. Treatment with NVP-BEZ235 also resulted in inhibition of tumor growth, which became significant after 14, 21 and 28 days (Fig. 4A). A statistically significant inhibition of tumor weight was also observed (Fig. 4B and C, left panel). No macroscopic metastases and ascites were observed in mice following sacrifice at day 28 , thus suggesting that the increase in body weight after week one in NVP-BEZ235-treated mice was due to natural growth (data not shown). To validate the results of the in vivo xenograft model, the effects of NVP-BEZ235 on sIL-2R (24) and sCD30 (25) were examined using serum samples obtained from SCID mice. The serum level of sIL-2R and sCD30 in the treated mice was lower than that of the control mice at 4 weeks after initiation of therapy, albeit statistically insignificant (Fig. 4C, middle and right panels). Finally, H\&E staining of tumor sections showed apoptosis of tumor cells, which was identified by condensation of the cytoplasm, fragmentation of the cell nuclei, and hyperchromatism and condensation of the chromatin (Fig. 4D, left panel). Furthermore, TUNEL staining identified numerous apoptotic cells in the tumors of the NVP-BEZ235-treated group (Fig. 4D, right panel).

\section{Discussion}

The PI3K/Akt/mTOR pathway plays an important role in cell proliferation, and is thus an interesting target for the treatment of various cancers, including hematological malignancies (26). Loss of function of phosphatases, most notably PTEN, which
Table I. The $\mathrm{IC}_{50}$ values for HTLV-1-infected T-cell lines and PBMC treated with NVP-BEZ235 and NVP-BKM120 for $48 \mathrm{~h}$.

\begin{tabular}{lcc}
\hline Cell line & NVP-BEZ235 $(\mathrm{nM})$ & NVP-BKM120 $(\mathrm{nM})$ \\
\hline MT-2 & 40.1 & 672.3 \\
MT-4 & 293.0 & 616.3 \\
SLB-1 & 178.3 & $2,248.6$ \\
HUT-102 & 11.9 & 397.2 \\
TL-OmI & 29.5 & $2,221.6$ \\
ED-40505(-) & 54.6 & $1,117.2$ \\
PBMC & $>15,000$ & $>6,000$ \\
\hline
\end{tabular}

HTLV-1, human T-cell leukemia virus type 1; PBMC, peripheral blood mononuclear cells.

regulate this pathway, has been demonstrated in ATL (6). It has been demonstrated that inhibition of signaling through mTORC1 inhibits growth of ATL cells (22). Although preclinical studies have reported that mTOR inhibitors can suppress tumor cell proliferation, monotherapy using these agents has not been as efficacious as initially expected (27). In this regard, mTORC1 inhibition can result in mTORC2-induced phosphorylation of Akt on Ser473 based on loss of the negative feedback loop through S6K, in addition to further activation of PI3K (13). Based on this background, we hypothesized that dual PI3K/mTOR inhibition could be superior to mTORC1 inhibition alone. In this study, we compared the efficacy of NVP-BEZ235 (a dual ATP-competitive PI3K/mTOR inhibitor), with RAD001 (a rapamycin analogue and specific inhibitor of mTORC1), and NVP-BKM120 (an ATP-competitive pan-PI3K inhibitor), in HTLV-1-infected T-cell lines.

Our results showed that RAD001 suppressed the growth of all HTLV-1-infected T-cell lines and that only low concentrations were required to inhibit the proliferation of sensitive cell lines; higher doses of RAD001 did not augment its efficacy. These findings suggest that effective anti-ATL protocols should include other compounds, in addition to RAD001. In contrast, treatment of HTLV-1-infected T-cell lines with NVP-BEZ235 and NVP-BKM120 led dose-dependently to a much larger decrease in cell growth. Based on the $\mathrm{IC}_{50}$ value of NVP-BEZ235 within the nanomolar range, NVP-BEZ235 was more potent in terms of growth inhibition than the selective inhibitor of PI3K. The poor anti-ATL activity of NVP-BKM235 and RAD001 at nanomolar concentrations might be due to their inability to block mTORC1 and mTORC2. Another potential factor is the limited suppressive effect of allosteric inhibitor on mTORC1, resulting in compensatory activation of feedback loops. However, NVP-BEZ235 downregulated phosphorylated PDK1 and Akt expression and downstream signaling of mTOR (phospho-4E-BP1 and phospho-p70-S6K).

We also showed that NVP-BEZ235 induced cell cycle arrest at the $\mathrm{G}_{0} / \mathrm{G}_{1}$ phase and slightly activated the apoptotic pathways. NVP-BEZ235 treatment inhibited $\mathrm{pRb}$ phosphorylation and cyclin D1, D2, E and CDK2, 4, 6 expressions in cells; consequently, it is highly possible that NVP-BEZ235 treatment causes $\mathrm{G}_{0} / \mathrm{G}_{1}$ arrest by blocking cyclin D-CDK4/6 and cyclin E-CDK2 complexes-mediated 
A
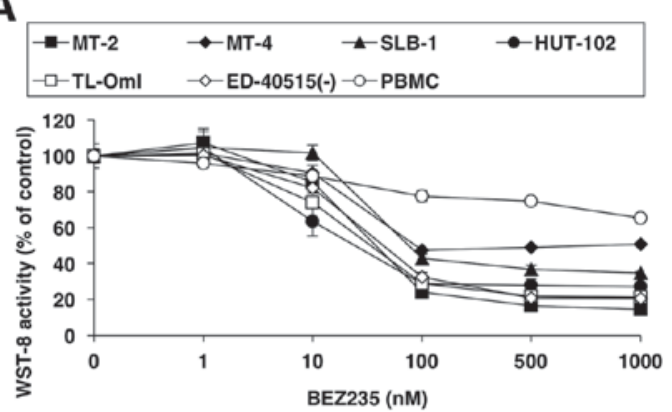

C
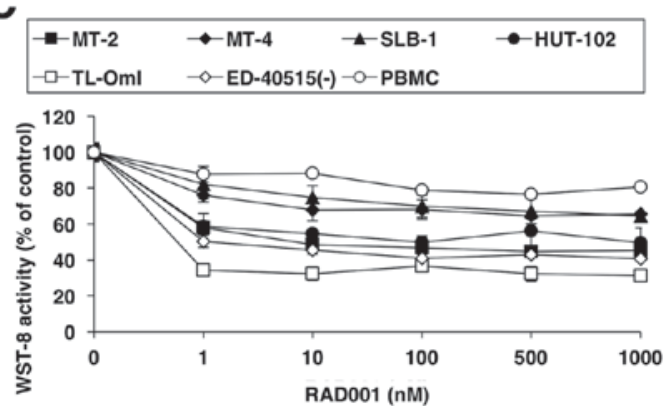

B

\begin{tabular}{|c|c|c|}
\hline$-\mathrm{m}-\mathrm{MT}-2$ & $\rightarrow$-SLB-1 & $\bullet-$ HUT-102 \\
\hline$-\square-T L-O m l$ & $\multimap$ ED-40515(-) - -PBMC & \\
\hline
\end{tabular}

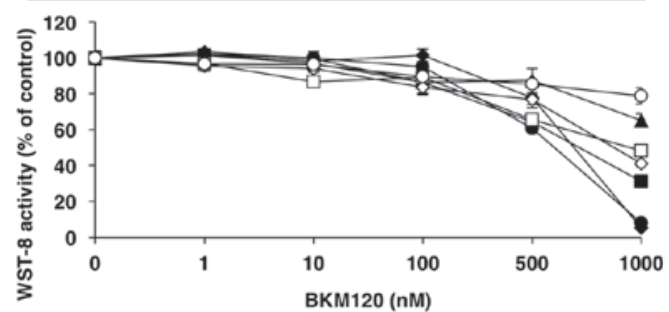

D

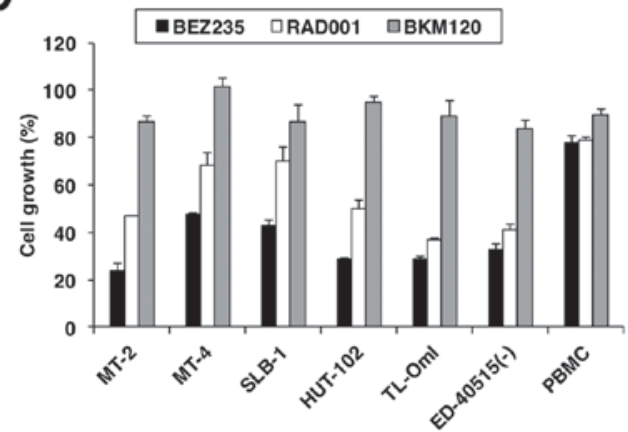

Figure 1. Effects of PI3K and mTOR inhibitors on cell proliferation. Several types of HTLV-1-infected T-cell lines and PBMC from a healthy donor were incubated with increasing concentrations of (A) NVP-BEZ235, (B) NVP-BKM120 and (C) RAD001 for 48 h. Cell proliferation was assessed with WST-8 assay. (D) Comparative analysis of the anti-ATL effect of PI3K and mTOR inhibitors. The indicated cells were treated with 100 nM of NVP-BEZ235, NVP-BKM120 and RAD001 for $48 \mathrm{~h}$ and cell proliferation was measured by WST-8 assay. Data were normalized to control values and the mean \pm standard deviation values of triplicate experiments are shown. PI3K, phosphatidylinositol 3-kinase; mTOR, mammalian target of rapamycin; HTLV-1, human T-cell leukemia virus type 1; PBMC, peripheral blood mononuclear cells.

A
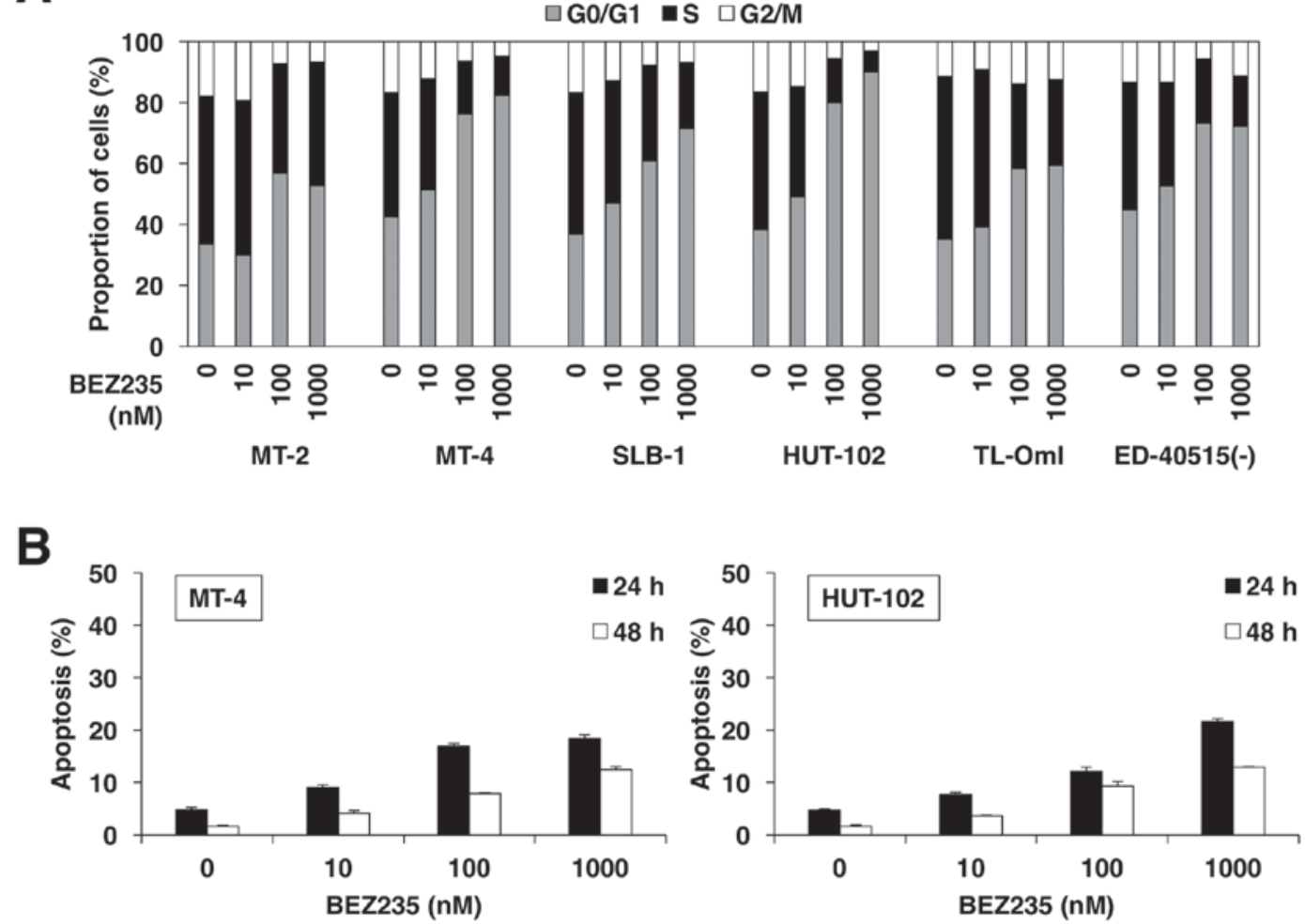

Figure 2. Effects of NVP-BEZ235 on cell cycle and cell death. (A) Cell cycle analysis of HTLV-1-infected T-cell lines treated with NVP-BEZ235. The indicated HTLV-1-infected T-cell lines were incubated with increasing concentrations of NVP-BEZ235 for $24 \mathrm{~h}$. The treated cells were stained with propidium iodide and analyzed using flow cytometry. Data are mean of triplicate experiments. (B) NVP-BEZ235 induces apoptosis. MT-4 and HUT-102 cells were exposed to the indicated concentrations of NVP-BEZ235 for 24 and $48 \mathrm{~h}$. Apoptotic cells were quantified by APO2.7 staining and flow cytometry. Data are mean \pm standard deviation percentages of positive cells from three experiments. HTLV-1, human T-cell leukemia virus type 1. 
A

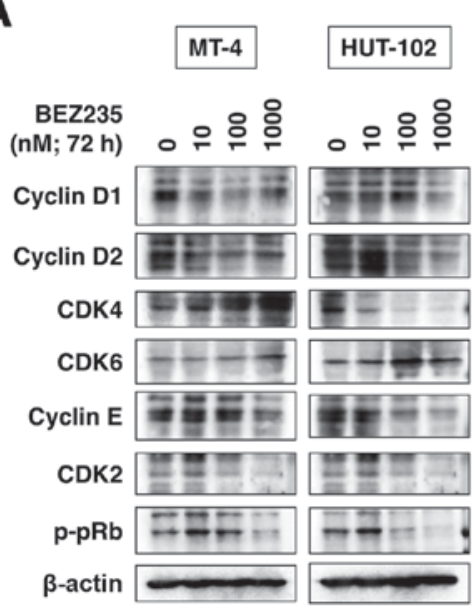

B

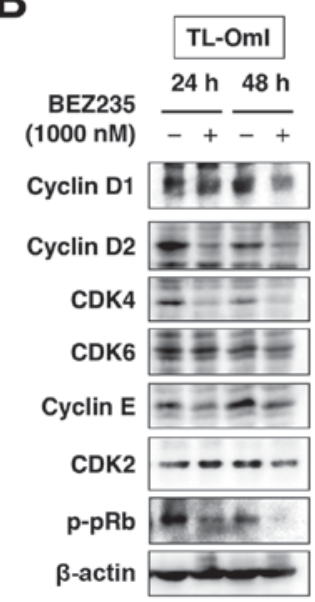

C

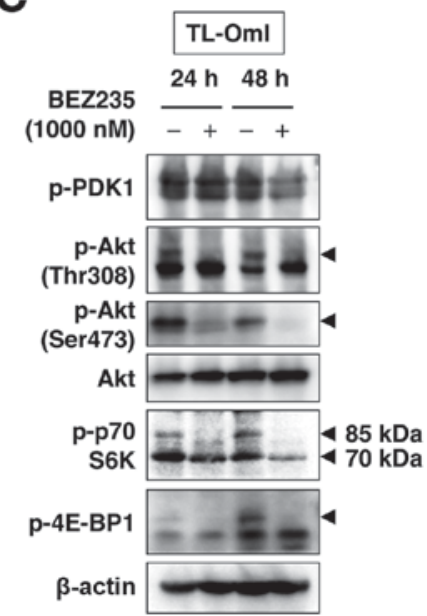

Figure 3. Expression of cell cycle regulatory proteins and downstream targets of the PI3K/mTOR pathway in HTLV-1-infected T-cell lines. (A) Western blots of cellular extracts from MT-4 and HUT-102 cells treated with increasing concentrations of NVP-BEZ235 for $72 \mathrm{~h}$. Cellular extracts were probed with antibodies against cell cycle-related markers. (B and C) Immunoblot analysis of extracts harvested from TL-OmI cells treated with the highest concentration of NVP-BEZ235. Membranes were probed with (B) antibodies against cell cycle regulatory proteins and (C) downstream targets of PI3K/mTOR pathway. Membranes were also probed with anti-actin antibody, as an internal loading control. PI3K, phosphatidylinositol 3-kinase; mTOR, mammalian target of rapamycin; HTLV-1, human T-cell leukemia virus type 1.
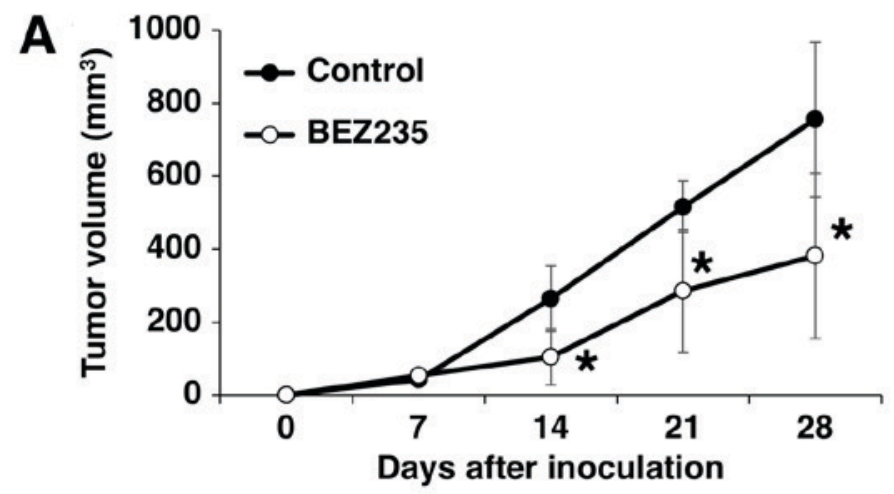

B
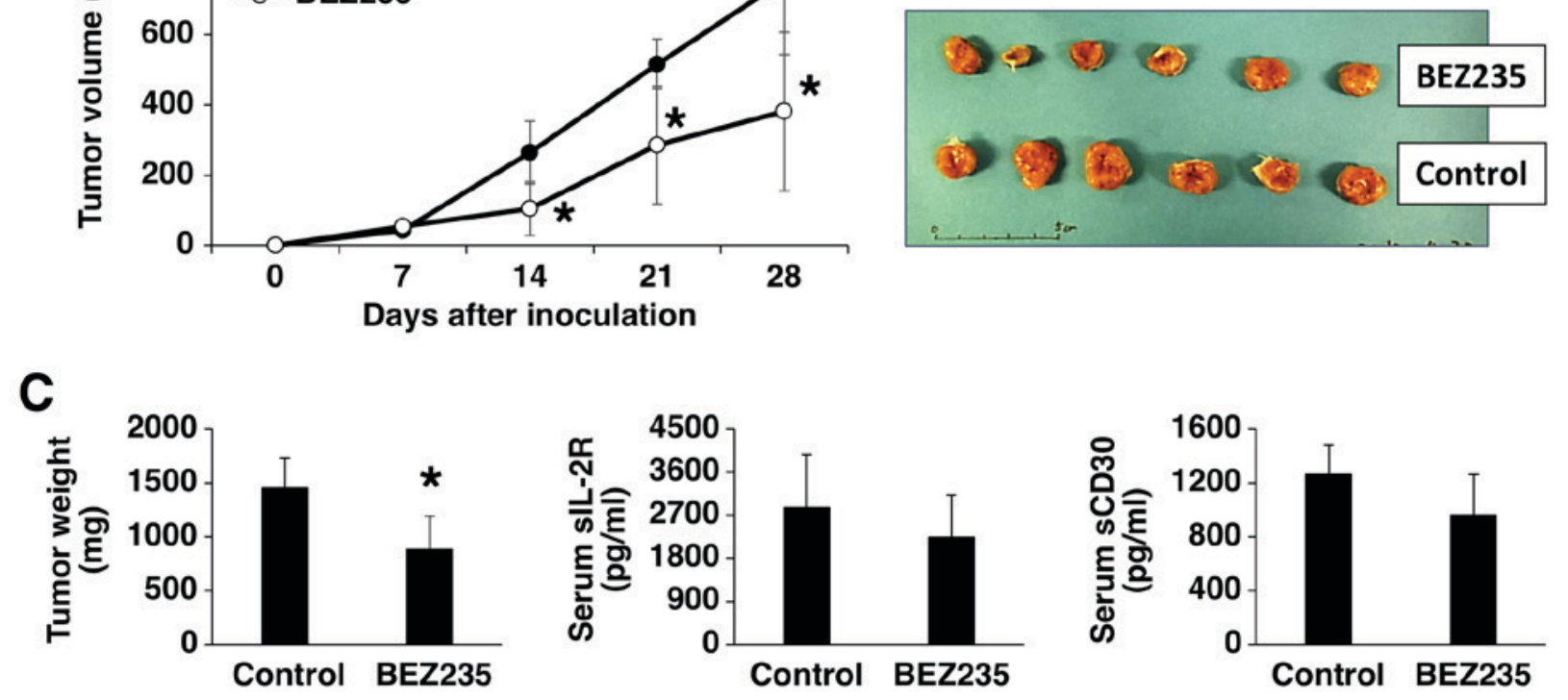

D
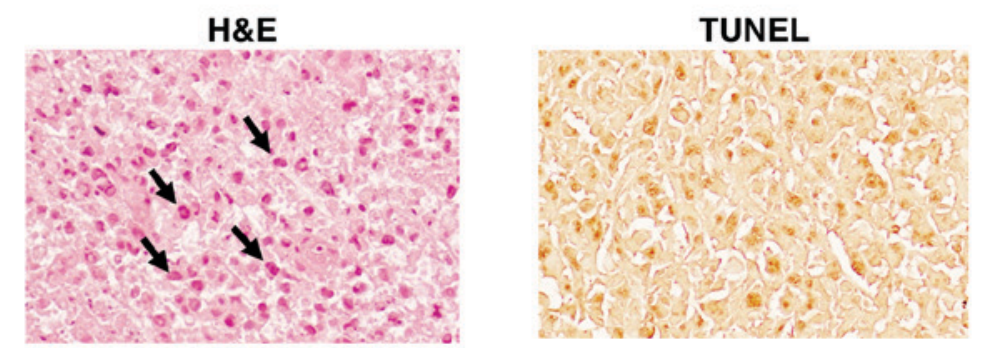

Figure 4. Effects of NVP-BEZ235 in experimental ATL rumor model. (A-C) Tumor progression is delayed in mice treated with NVP-BEZ235 administered by oral gavage. HUT-102 tumor bearing mice were treated with either NVP-BEZ235 at a dose of $40 \mathrm{mg} / \mathrm{kg}$ body weight or vehicle for 28 days. (A) Tumor volume after 4 weeks of daily treatment with NVP-BEZ235. Tumor volume was measured with a caliper during the course of the experiment. (B) NVP-BEZ235-treated mice had smaller tumors. Three hours after the last dose, the mice were sacrificed and tumor was removed and analyzed. (C) Tumor weight (left panel) was measured, and concentrations of serum sIL-2R (middle panel) and SCD30 (right panel) were determined by ELISA. Data are mean \pm standard deviation for each group of mice $(n=6)$. "P<0.05, compared with the control group. (D) Histopathological examination of H\&E- and TUNEL-stained HUT-102 xenograft tumor tissues from a representative mouse treated with NVP-BEZ235. Arrows show apoptotic cells. Magnification, $\mathrm{x} 800$. 
$\mathrm{pRb}$ phosphorylation, which is critical to the progression of the cell cycle at the $G_{0} / G_{1}$ to $S$ transition. The effectiveness of this agent was confirmed in xenograft models of ATL. NVP-BEZ235 markedly suppressed tumor growth in these mice compared with control group. To our knowledge, this is the first study to show that the novel dual PI3K/mTOR inhibitor NVP-BEZ235 is effective against ATL, both in vitro and in vivo. Therapies targeting the PI3K/Akt/mTOR pathway may therefore be of interest for ATL and we believe that they are promising candidates for ATL therapy.

In conclusion, simultaneous inhibition of PI3K, mTORC1 and mTORC2 by the dual inhibitor NVP-BEZ235 showed profound anti-ATL activity. Our data provide a strong preclinical rationale for phase I clinical studies to test this compound in patients with ATL.

\section{Acknowledgements}

The authors thank Dr Michiyuki Maeda for ED-40515(-), the Fujisaki Cell Center, Hayashibara Biochemical Laboratories, Inc. (Okayama, Japan) for HUT-102, and Novartis Institutes for BioMedical Research (Basel, Switzerland) for the kind supply of NVP-BEZ235, NVP-BKM120 and RAD001. This study was supported in part by two grants from JSPS KAKENHI (nos. 15K18414 and 25461428).

\section{References}

1. Yoshida M, Seiki M, Yamaguchi K and Takatsuki K: Monoclonal integration of human T-cell leukemia provirus in all primary tumors of adult T-cell leukemia suggests causative role of human T-cell leukemia virus in the disease. Proc Natl Acad Sci USA 81: 2534-2537, 1984

2. Vose J, Armitage J and Weisenburger D; International T-Cell Lymphoma Project: International peripheral T-cell and natural killer/T-cell lymphoma study: Pathology findings and clinical outcomes. J Clin Oncol 26: 4124-4130, 2008.

3. Ishida T, Hishizawa M, Kato K, Tanosaki R, Fukuda T, Taniguchi S, Eto T, Takatsuka Y, Miyazaki Y, Moriuchi Y, et al: Allogeneic hematopoietic stem cell transplantation for adult T-cell leukemia-lymphoma with special emphasis on preconditioning regimen: A nationwide retrospective study. Blood 120: 1734-1741, 2012.

4. Rosich L, Saborit-Villarroya I, López-Guerra M, Xargay-Torrent S, Montraveta A, Aymerich M, Villamor N, Campo E, Pérez-Galán P, Roué $\mathrm{G}$ and Colomer D: The phosphatidylinositol-3-kinase inhibitor NVP-BKM120 overcomes resistance signals derived from microenvironment by regulating the Akt/FoxO3a/Bim axis in chronic lymphocytic leukemia cells. Haematologica 98: 1739-1747, 2013.

5. Liu P, Cheng H, Roberts TM and Zhao JJ: Targeting the phosphoinositide 3-kinase pathway in cancer. Nat Rev Drug Discov 8: 627-644, 2009.

6. Nakahata S, Ichikawa T, Maneesaay P, Saito Y, Nagai K, Tamura T, Manachai N, Yamakawa N, Hamasaki M, Kitabayashi I, et al: Loss of NDRG2 expression activates PI3K-AKT signalling via PTEN phosphorylation in ATLL and other cancers. Nat Commun 5: 3393, 2014

7. Rodon J, Dienstmann R, Serra V and Tabernero J: Development of PI3K inhibitors: Lessons learned from early clinical trials. Nat Rev Clin Oncol 10: 143-153, 2013.

8. Zheng Y, Yang J, Qian J, Zhang L, Lu Y, Li H, Lin H, Lan Y, Liu Z, He J, et al: Novel phosphatidylinositol 3-kinase inhibitor NVP-BKM120 induces apoptosis in myeloma cells and shows synergistic anti-myeloma activity with dexamethasone. J Mol Med (Berl) 90: 695-706, 2012.

9. Ren H, Zhao L, Li Y, Yue P, Deng X, Owonikoko TK, Chen M, Khuri FR and Sun SY: The PI3 kinase inhibitor NVP-BKM120 induces GSK3/FBXW7-dependent Mcl-1 degradation, contributing to induction of apoptosis and enhancement of TRAIL-induced apoptosis. Cancer Lett 338: 229-238, 2013.
10. Zang C, Eucker J, Liu H, Coordes A, Lenarz M, Possinger K and Scholz CW: Inhibition of pan-class I phosphatidyl-inositol-3-kinase by NVP-BKM120 effectively blocks proliferation and induces cell death in diffuse large B-cell lymphoma. Leuk Lymphoma 55: 425-434, 2014.

11. Rodon J, Braña I, Siu LL, De Jonge MJ, Homji N, Mills D, Di Tomaso E, Sarr C, Trandafir L, Massacesi C, et al: Phase I dose-escalation and -expansion study of buparlisib (BKM120), an oral pan-Class I PI3K inhibitor, in patients with advanced solid tumors. Invest New Drugs 32: 670-681, 2014.

12. Bhat M, Robichaud N, Hulea L, Sonenberg N, Pelletier J and Topisirovic I: Targeting the translation machinery in cancer. Nat Rev Drug Discov 14: 261-278, 2015.

13. O'Reilly KE, Rojo F, She QB, Solit D, Mills GB, Smith D, Lane H, Hofmann F, Hicklin DJ, Ludwig DL, et al: mTOR inhibition induces upstream receptor tyrosine kinase signaling and activates Akt. Cancer Res 66: 1500-1508, 2006.

14. Bhatt AP, Bhende PM, Sin SH, Roy D, Dittmer DP and Damania B: Dual inhibition of PI3K and mTOR inhibits autocrine and paracrine proliferative loops in PI3K/Akt/mTOR-addicted lymphomas. Blood 115: 4455-4463, 2010.

15. Maira SM, Stauffer F, Brueggen J, Furet P, Schnell C, Fritsch C, Brachmann $\mathrm{S}$, Chène $\mathrm{P}$, De Pover $\mathrm{A}$, Schoemaker $\mathrm{K}$, et al: Identification and characterization of NVP-BEZ235, a new orally available dual phosphatidylinositol 3-kinase/mammalian target of rapamycin inhibitor with potent in vivo antitumor activity. Mol Cancer Ther 7: 1851-1863, 2008.

16. Bhende PM, Park SI, Lim MS, Dittmer DP and Damania B: The dual PI3K/mTOR inhibitor, NVP-BEZ235, is efficacious against follicular lymphoma. Leukemia 24: 1781-1784, 2010.

17. Shortt J, Martin BP, Newbold A, Hannan KM, Devlin JR, Baker AJ, Ralli R, Cullinane C, Schmitt CA, Reimann M, et al: Combined inhibition of PI3K-related DNA damage response kinases and mTORC1 induces apoptosis in MYC-driven B-cell lymphomas. Blood 121: 2964-2974, 2013.

18. Chiarini F, Grimaldi C, Ricci F, Tazzari PL, Evangelisti C, Ognibene A, Battistelli M, Falcieri E, Melchionda F, Pession A, et al: Activity of the novel dual phosphatidylinositol 3-kinase/mammalian target of rapamycin inhibitor NVP-BEZ235 against T-cell acute lymphoblastic leukemia. Cancer Res 70: 8097-8107, 2010.

19. Ishiyama M, Miyazono Y, Sasamoto K, Ohkura Y and Ueno K: A highly water-soluble disulfonated tetrazolium salt as a chromogenic indicator for NADH as well as cell viability. Talanta 44: 1299-1305, 1997.

20. Zhang C, Ao Z, Seth A and Schlossman SF: A mitochondrial membrane protein defined by a novel monoclonal antibody is preferentially detected in apoptotic cells. J Immunol 157: 3980-3987, 1996.

21. Tomayko MM and Reynolds CP: Determination of subcutaneous tumor size in athymic (nude) mice. Cancer Chemother Pharmacol 24: 148-154, 1989

22. Ikezoe T, Nishioka C, Bandobashi K, Yang Y, Kuwayama Y, Adachi Y, Takeuchi T, Koeffler HP and Taguchi H: Longitudinal inhibition of PI3K/Akt/mTOR signaling by LY294002 and rapamycin induces growth arrest of adult T-cell leukemia cells. Leuk Res 31: 673-682, 2007.

23. Weinberg RA: The retinoblastoma protein and cell cycle control. Cell 81: 323-330, 1995.

24. Kamihira S, Atogami S, Sohda H, Momita S, Yamada Y and Tomonaga M: Significance of soluble interleukin-2 receptor levels for evaluation of the progression of adult T-cell leukemia. Cancer 73: 2753-2758, 1994

25. Nishioka C,TakemotoS,KataokaS, YamanakaS,Moriki T,ShodaM, Watanabe T and Taguchi H: Serum level of soluble CD30 correlates with the aggressiveness of adult T-cell leukemia/lymphoma. Cancer Sci 96: 810-815, 2005.

26. Martelli AM, Chiarini F, Evangelisti C, Cappellini A, Buontempo F, Bressanin D, Fini M and McCubrey JA: Two hits are better than one: Targeting both phosphatidylinositol 3-kinase and mammalian target of rapamycin as a therapeutic strategy for acute leukemia treatment. Oncotarget 3: 371-394, 2012.

27. Huang Z, Wu Y, Zhou X, Qian J, Zhu W, Shu Y and Liu P: Clinical efficacy of mTOR inhibitors in solid tumors: A systematic review. Future Oncol 11: 1687-1699, 2015. 